\title{
Gender as a factor affecting NK cell activity in patients successfully treated for chronic hepatitis $\mathbf{C}$ with direct-acting antivirals
}

\author{
AGATA ZIENTARSKA ${ }^{1}$, JUSTYNA MIKUŁA-PIETRASIK ${ }^{2}$, MARIUSZ KACZMAREK ${ }^{3,4,5}$, \\ ALEKSANDRA WITKOWSKA ${ }^{1}$, BŁAŻEJ ROZPŁOCHOWSKI ${ }^{1}$, ARLETA KOWALA-PIASKOWSKA ${ }^{1}$, \\ KRZYSZTOF KSIĄŻEK ${ }^{2}$, JAN ŻEROMSKI ${ }^{3}$, IWONA MOZER-LISEWSKA ${ }^{l}$
}

\author{
${ }^{1}$ Chair and Department of Infectious Diseases, Hepatology and Acquired Immunodeficiencies, Karol Marcinkowski University of Medical \\ Sciences, Poznan, Poland \\ ${ }^{2}$ Department of Pathophysiology of Ageing and Civilization Diseases, Karol Marcinkowski University of Medical Sciences, Poznan, Poland \\ ${ }^{3}$ Chair of Clinical Immunology, Karol Marcinkowski University of Medical Sciences, Poznan, Poland \\ ${ }^{4}$ Department of Cancer Immunology, Karol Marcinkowski University of Medical Sciences, Poznan, Poland \\ ${ }^{5}$ Department of Diagnostics and Cancer Immunology, Greater Poland Cancer Centre, Poznan, Poland
}

\begin{abstract}
Chronic hepatitis $\mathrm{C}(\mathrm{CHC})$ affects the activity of natural killer (NK) cells, but successful interferonfree treatment partially restores it. The goal of this study was to assess whether gender influences NK functionality. We examined 21 post-menopausal women and 24 men with $\mathrm{CHC}$ who were treated with direct-acting antivirals (DAA) and 33 healthy volunteers. Using flow cytometry, we analysed KIR2DS4, NKG2D, NKp30, KIR2DL2/DL3, NKG2A and TRAIL on the surface of NK cells. Intracellular granzyme B was also assessed and serum CXCL10 was quantified via ELISA. Overall, patients with CHC had higher expression of KIR2DS4, NKG2A, and NKp30 relative to the control group. Further, CHC patients had a lower percentage of $N K$ cells among lymphocytes relative to the control group. After treatment, KIR2DS4, KIR2DL2/DL, NKG2A, TRAIL and NKp30 on NK cells were decreased whilst the percentage of $N K$ cells and the expression of granzyme $B$ and NKG2D increased. Prior to treatment, serum CXCL1O was elevated, but it was inhibited post-treatment. We observed gender-specific differences in the expression of KIR2DL2/DL3 (higher in women) and NKp30 (elevated in men) compared to CHC/control groups. After treatment, KIR2DL2/DL3, NKp3O and CXCL10 dropped only in the female group while granzyme B increased in the male group. In conclusion, the response of NK cells among men and women of post-menopausal ages with CHC differs. Our research may lead to more studies on the different nature of female and male immune systems in the context of HCV infection and treatment.
\end{abstract}

Key words: HCV, gender, NK cells, direct-acting antivirals.

(Cent Eur J Immunol 2021; 46 (4): 481-491)

\section{Introduction}

Natural killer (NK) cells are a crucial element of the host's innate immune response to viral infections. The protective and effector functions of NK cells involve two strategies. Firstly, they induce cell death through the controlled release of cytotoxic granules containing perforin and granzymes or by inducing death receptor-mediated apoptosis, which is facilitated by the expression of Fas ligand or TNF-related apoptosis-inducing ligand (TRAIL) [1]. Secondly, NK cells can produce pro-inflammatory and immunosuppressive cytokines as well as chemokines which amplify and facilitate the success of the initial host immune response during pathogen challenge [2].
NK cells do not possess antigen-specific receptors, nor are they activated by antigen presentation. Rather, NK activation is regulated by several inhibitory or activating surface receptors on the NK cell surface and their ligands on target cells. On healthy cells, major histocompatibility complex (MHC) class I molecules prevent the mobilization and cytotoxic action of NK cells via the presence of inhibitory KIR receptors. Virus-infected cells usually have decreased expression of MHC class I and decreased presentation of stimulating ligands [3].

Chronic hepatitis $\mathrm{C}$ (CHC) does not lead to increased activation of NK cells; however, $\mathrm{CHC}$ has been shown to dysregulate NK cell functionality [4]. The expression of particular activating and inhibiting receptors on NK cells

Correspondence: Agata Zientarska, Chair and Department of Infectious Diseases, Hepatology and Acquired Immunodeficiencies, Karol Marcinkowski University of Medical Sciences, Poznan, e-mail: agatakierepa@gmail.com Submitted: 17.03.2021; Accepted: 05.07.2021 
is distorted; nevertheless, authors have reported various results of their studies [5-17].

Female and male immune systems differ in many aspects. However, the main differences underpinning sexual dimorphism are hormonal and genetic. Females develop enhanced innate and adaptive immune responses compared to males, which makes them less predisposed to various infections and malignancies, but more susceptible to autoimmune diseases [18]. Additionally, males have a higher count of total NK cells, a higher frequency of CD56 $6^{\mathrm{dim}} \mathrm{NK}$ cells and higher expression of CD57, which is found on NK and $\mathrm{T}$ cell surfaces [19]. In women of reproductive age, oestrogen inhibits NK cell cytotoxicity at the ovulatory phase [20]. However, women over the age of 70 have more vigorous NK cell cytotoxic activity relative to men [21]. Among chronically hepatitis B virus (HBV)-infected patients, intrahepatic NK cells have higher degranulation activity in women and correlate with the oestradiol level [22].

Interestingly, gender disparity has an impact on the prognosis in CHC infection. Immunological differences combine with hepatoprotective effects of oestradiol and oestrogen [23]. As a result, in hepatitis C virus (HCV)-associated cirrhosis, male patients with hepatocellular carcinoma (HCC) have a worse clinical outcome relative to women [24]. Further, short HCV infections treated with interferon-based therapies had a higher chance of success in females [25]. Additionally, among $\mathrm{HCV}$-infected patients treated with direct-acting antivirals (DAA), the rate of HCC recurrence and occurrence is higher among men [26].

Together, these lines of evidence suggest that gender-specific effects may have a significant effect on disease outcome and treatment. This study aimed to investigate the influence of gender on changes observed in NK cells of chronically $\mathrm{HCV}$-infected patients who were successfully treated with novel, interferon-free therapies.

\section{Material and methods}

\section{Ethical statement}

This study was carried out in accordance with the Declaration of Helsinki of the World Medical Association and was approved by the Ethical Committee of Poznan University of Medical Sciences.

\section{Study design}

Patients who were treated at the Department of Infectious Diseases, Hepatology and Acquired Immunodeficiencies of Poznan University of Medical Sciences were inducted into this study. The final cohort consisted of 45 patients with $\mathrm{CHC}$ genotype $1 \mathrm{~b}$ and qualified for DAA treatment (21 females and 24 males) and had differing stages of liver fibrosis. To eliminate the impact of rapid hormonal changes in the course of the menstrual cycle, only post-menopausal women were included. The exclusion criteria were as follows: the presence of $\mathrm{HCC}$ or any other malignancy, human immunodeficiency virus (HIV) co-infection, presence of HBsAg, any active infection (other than HCV), and the current administration of any immunomodulatory or immunosuppressive drugs.

Patients were tested up to 7 days before the treatment (T0) and twice after the completed regimen - an average of 200 days after the first dose (T1) and 167 days later (T2). The treatment regimens for all patients were as follows: 16 patients were treated with sofosbuvir/ledipasvir + ribavirin ( 8 females and 8 males); 7 patients were treated with sofosbuvir/ledipasvir only ( 2 females and 5 males); 7 patients who received regimens consisting of ombitasvir/ paritaprevir/ritonavir + dasabuvir (2 female and 5 males); 4 patients who received grazoprevir/elbasvir ( 2 female and 2 males); 8 who received sofosbuvir/velpatasvir (4 females and 4 males); and 4 patients who received glecaprevir/parenthetic ( 3 females and 1 male). All treatments regimens were prescribed in accordance with Polish recommendations [27]. All patients completed the therapy and achieved a sustained virological response (SVR). A group consisting of 33 volunteers without $\mathrm{HCV}$ infection (22 men and 11 women) of post-menopausal age, meeting the same exclusion criteria as the study group, served as the control group. 16 subjects within the control group had a history of excess alcohol intake but adhered to a six-month abstinence period before the study. Some of these participants included individuals with hepatic fibrosis due to alcohol-related liver disease. They did not display any evidence of other types of liver diseases.

\section{Liver pathology assessment}

Liver stiffness was quantified using the transient elastography technique, using the FibroScan Compact device (Echosens, Paris, France) in compliance with the manufacturer's instructions [28].

\section{Flow cytometry}

Cell immunophenotyping was performed by flow cytometry using the direct fluorescence method, which we described in our previous study [29]. Briefly, the appropriate fluorochrome-labelled monoclonal antibodies (described in Table 1) were added to the cytometric tubes at a rate of $5 \mu \mathrm{l}$ each. $100 \mu \mathrm{l}$ of peripheral blood previously mixed with an anticoagulant solution (EDTA) in vacutainer tubes was added to the test tubes. Samples were mixed gently by vortex and incubated for $15 \mathrm{~min}$ at room temperature and protected from light. After this time, $500 \mu \mathrm{l}$ of erythrocyte lysis buffer (Becton Dickinson) was added to the test tubes and the tubes were incubated for $10 \mathrm{~min}$. Lysis was stopped by adding phosphate-buffered saline (PBS) solution. Samples were then centrifuged at $1500 \mathrm{rpm}$ for $5 \mathrm{~min}$ at room temperature to separate residual lysed erythrocytes, plasma proteins, and other morphotic elements of blood from leukocytes. The supernatants were discarded. This step was repeated twice. Granzyme B was studied intracellularly after the additional permeabilisation of cells 
Table 1. Antibodies used in the study for cell immunophenotyping

\begin{tabular}{lccc}
\hline Antibody & Fluorochrome & Clone & Source \\
\hline CD3 & PerCP & SK7 & BD Biosciences \\
\hline CD16 & FITC & NKP15 & BD Biosciences \\
\hline CD56 & PE-Cy 7 & NCAM16.2 & BD Biosciences \\
\hline CD158b (KIR2DL2/DL3) & APC & DX27 & Miltenyi Biotec \\
\hline CD158i (KIR2DS4) & PE & JJC11.6 & Miltenyi Biotec \\
\hline CD159a (NKG2A) & PE & REA110 & Miltenyi Biotec \\
\hline CD314 (NKG2D) & APC & BAT221 & Miltenyi Biotec \\
\hline CD337 (NKp30) & APC & AF29-4D12 & Miltenyi Biotec \\
\hline CD253 (TRAIL) & PE & RIK-2.1 & BD Pharmingen \\
\hline Granzyme B & PE & GB11 & BD Pharmingen \\
\hline
\end{tabular}

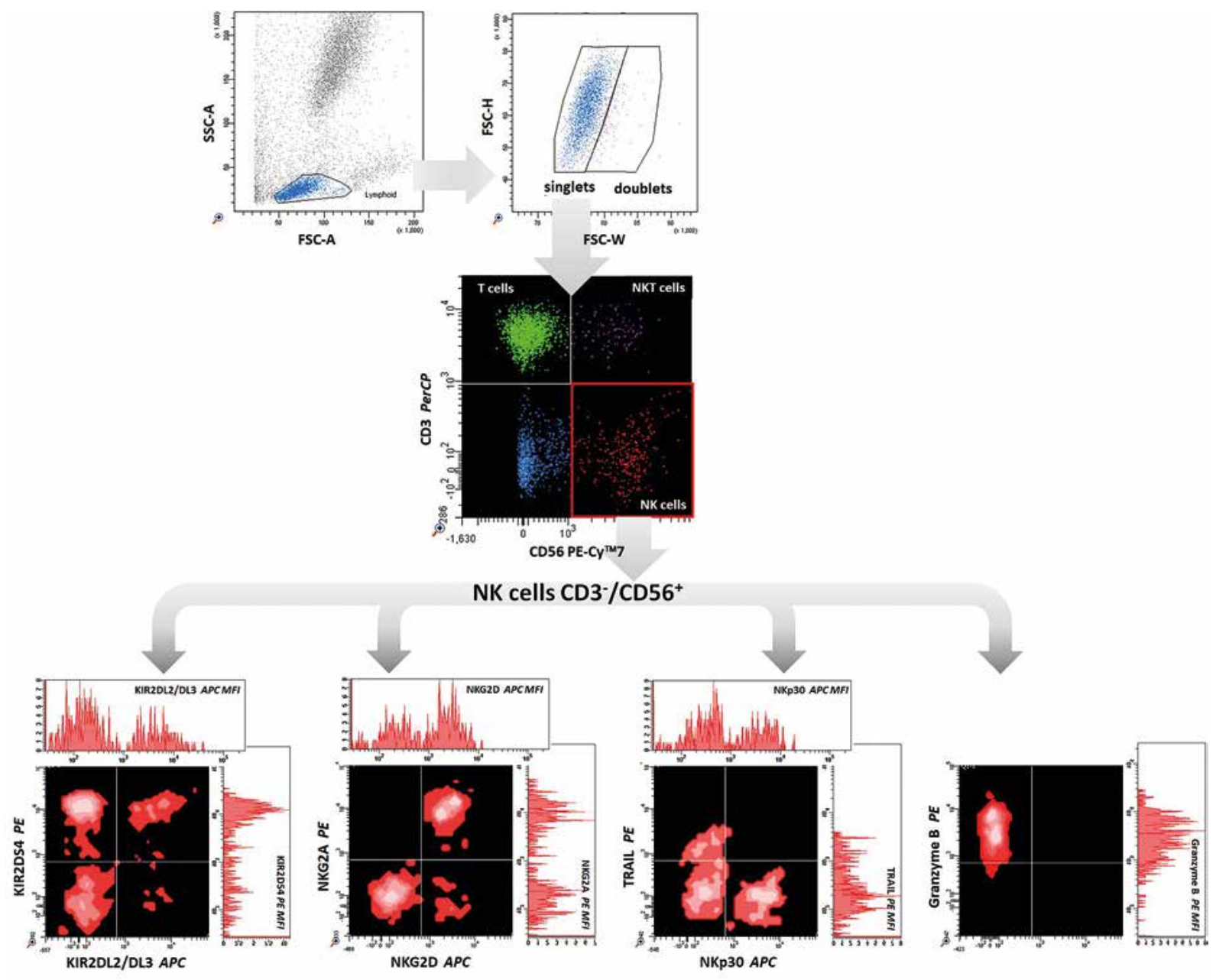

Fig. 1. Gating strategy for cytometric evaluation of NK cells. Percentage and histogram for mean fluorescence intensity (MFI) of the tested antigens. Graphs are from BD FACSDiva Software v. 6.1.2 Firmware v. 1.8 (BD FACSAria)

with Perm/Wash buffer (BD Biosciences). The cell pellets were resuspended in $200 \mu \mathrm{l}$ of PBS. The samples were then acquired using a FACS Canto flow cytometer (Becton Dickinson). The obtained results were analysed with the
FACS Diva software (Becton Dickinson), integrated with the cytometer. For each examined antibody, the percentage of positive cells and mean fluorescence intensity (MFI) were determined (Fig. 1). 


\section{ELISA}

The serum concentration of human CXCL10/IP-10 was measured using the appropriate DuoSet Immunoassay Development kits (R\&D Systems, Minneapolis, USA), in accordance with the manufacturer's instructions.

\section{Statistical analysis}

Statistical analyses were conducted using the STATISTICA software version 13.0 (StatSoft, Cracow, Poland). As the Shapiro-Wilk test indicated that the data from most variables were not normally distributed, non-parametric tests were used; dependent variables were analysed with the Wilcoxon signed-rank test and non-dependent variables were analysed with the Mann-Whitney $U$ test. A $p$-value $\leq 0.05$ was considered statistically significant [30].

\section{Results}

Among the patients who met the inclusion criteria, $66.7 \%$ had radiological and biochemical indicators of liver cirrhosis which were confirmed by FibroScan. There were no significant differences regarding liver function, nor basic biochemical and morphological parameters between the genders (Table 2). After treatment, hepatic stiffness, alanine transaminase (ALT), aspartate transaminase (AST) and total bilirubin level were significantly decreased in all patients ( $p=0.002,0.00002,0.00002$ and 0.007 , respectively). Further, both the platelet (PLT) and white blood cell (WBC) counts were significantly increased ( $p=0.0004$ and 0.00002 , respectively). These changes were similar between genders.

There were significant changes in the expression of NK receptors and their MFI after the treatment. Further, the percentage of NK cells among all lymphocytes was significantly lower in the $\mathrm{CHC}$ group relative to the control group ( $p=0.004)$. However, treatment was observed to rescue the percentage of $\mathrm{NK}$ recruitment at both the $\mathrm{T} 1$ and $\mathrm{T} 2$ timepoints ( $p=0.01$ and 0.03 , respectively). Additionally, no differences in NK cell frequency were observed between the control group and the cured population.

We assessed three activating NK receptors: NKG2D, NKp30 and KIR2DS4. Our data indicate that only the expression of KIR2DS4 in patients with $\mathrm{CHC}(p=0.009)$ remained increased after the treatment ( $p=0.03$ at T1). MFI of NKp30 was significantly increased in patients with $\mathrm{CHC}$ relative to the control group $(p=0.04)$ and there was a visible but statistically insignificant increase in the MFI of KIR2DS4. After the treatment, the intensity of fluorescence of those receptors was decreased at T1 ( $p=0.007$ and 0.0005 , respectively), while at $\mathrm{T} 2$, diminished expression of KIR2DS4 was observed $(p=0.006)$.

Additionally, two inhibitory receptors present on the surface of NK cells, NKG2A and KIR2DL2/DL3 (CD158b), were analysed. No changes in the expression of NKG2A and KIR2DL2/DL3 (CD158b) on NK cells, regardless of $\mathrm{HCV}$ status, were observed. However, the MFI of NKG2A was significantly increased among CHC patients relative to the control group $(p=0.0006)$. CD158 was also elevated, but this increase was not statistically significant. After therapy, the MFI of both receptors was decreased ( $p=0.0004$ and 0.003 in T1, $p=0.0006$ and 0.03 at $\mathrm{T} 2$, respectively).

To assess the primary mechanisms of NK cell cytotoxicity, we measured the expression and MFI of the TRAIL protein and granzyme B. In the study group, an increase in granzyme B ( $p=0.02$ at T1) and a decrease of TRAIL expression on NK cells ( $p=0.04$ at T2) were observed after DAA treatment.

Finally, serum levels of the NK-chemoattractant chemokine C-X-C chemokine 10 (CXCL10) were analysed via ELISA. CXCL10 was significantly elevated among chronically HCV-infected patients $(p=0.001)$ and was decreased after therapy ( $p=0.001$ at T1 and 0.00003 at T2) (Table 3).

We then compared all the parameters detailed above between the male and female groups. The MFI of NKp30 was higher only in males (median 3209 vs. 2697, $p=0.016)$ and the MFI of KIR2DL2/DL3 was elevated in females (median 7272 vs. 4915, $p=0.02$ ) when comparing $\mathrm{CHC}$ patients to the control group. Furthermore, there were a few significant differences in NK cells during HCV eradication between men and women. These data are presented in the Figures 2-5.

\section{Discussion}

After successful DAA therapy, liver fibrosis was decreased and there was a significant improvement in biochemical and haematological parameters. These data are comparable to the changes presented in the literature [31]. Interferon-free $\mathrm{HCV}$ therapies are undoubtedly a great achievement of modern medicine.

Within the CHC patient group, several phenotypic changes in NK cells were partially restored after the eradication of the hepatitis $C$ virus. Generally, these phenotypic changes were consistent with those described in other studies (Table 4).

The expression of inhibitory receptors such as KIR2DL2/DL3 or NKG2A is largely determined by genetics [32]. Therefore, the dynamics of the receptor that we have described in this study are consistent with the results of other studies.

The upregulation or activation of receptor expression is environmentally influenced [32]. Thus, the diversity of expression of NKG2D and NKp30 observed in this study relative to previous studies on $\mathrm{HCV}$-infected patients can likely be attributed to environmental factors. Further, the expression profile observed in $\mathrm{CHC}$ patients relative to the control groups seems to be the essential factor that can be 
Table 2. Characteristics of the control group and the study group before the treatment (T0), then 6 months (T1) and one year (T2) after the beginning of DAA treatment. ALT - alanine transaminase, AST - aspartate transaminase. Presented $p$ values refer to differences between the control group and T0 ( $p 1$, Mann-Whitney $U$ test), then between T0 vs. T1 and T0 vs. T2 ( $p 2$ and $p 3$, respectively; Wilcoxon signed-rank test). In the Mann-Whitney $U$ test, there are no significant differences between females and males in the presented parameters. Data are median (interquartile range)

\begin{tabular}{|c|c|c|c|c|c|c|c|}
\hline Parameter & Control & T0 & T1 & $\mathbf{T 2}$ & $p 1$ & $p 2$ & $p 3$ \\
\hline Total number & 33 & 45 & 45 & 29 & - & - & - \\
\hline Female & 11 & 21 & 21 & 11 & - & - & - \\
\hline Male & 22 & 24 & 24 & 18 & - & - & - \\
\hline Age (years) & $47(31-65)$ & $62(56-68)$ & $62(56-68)$ & $62(51-70)$ & .003 & - & - \\
\hline Female & $52(44-60)$ & $66(59-69)$ & $66(59-69)$ & $66(56-70)$ & .004 & - & - \\
\hline Male & $38(30-66)$ & $60(46-65)$ & $60(46-65)$ & $61(44-67)$ & $>.05$ & - & - \\
\hline Estimated time of infection (years) & - & $37(33-41)$ & $37(33-41)$ & $36(32-42)$ & - & - & - \\
\hline Female & - & $37(34-43)$ & $37(34-43)$ & $39(34-43)$ & - & - & - \\
\hline Male & - & $36(31-40)$ & $36(31-40)$ & $35(28-39)$ & - & - & - \\
\hline $\begin{array}{l}\text { Number of patients with liver } \\
\text { cirrhosis }\end{array}$ & 7 & 30 & 30 & 15 & - & - & - \\
\hline Female & 2 & 15 & 15 & 6 & - & - & - \\
\hline Male & 5 & 15 & 15 & 9 & - & - & - \\
\hline Liver fibrosis $(\mathrm{kPa})$ & $6.0(4.3-12.0)$ & $24.8(8.5-36.3)$ & $16.2(6.8-27.4)$ & $9.0(6.2-23.3)$ & $<.001$ & .007 & $<.001$ \\
\hline Female & $5.5(4.3-12)$ & $24.8(8.7-45.4)$ & $15.3(6.8-26.7)$ & $11.6(6.8-25.4)$ & .005 & $>.05$ & .033 \\
\hline Male & $6.8(4.8-12.6)$ & $25.4(7.4-35.4)$ & $15.9(6.5-30.8)$ & $8.8(6.0-18.0)$ & $<.001$ & .029 & .004 \\
\hline White blood cells $\times 10^{3} \mathrm{WBC} / \mathrm{mm}^{3}$ & $6.5(5.7-7.0)$ & $5.0(4.3-6.2)$ & $6.1(5.1-7.3)$ & $6.0(5.5-7.7)$ & .001 & $<.001$ & .001 \\
\hline Female & $6.0(4.5-6.5)$ & $4.9(4.3-5.8)$ & $6.4(4.9-7.3)$ & $6.0(4.7-7.2)$ & .002 & .021 & $>.05$ \\
\hline Male & $6.8(5.7-8.0)$ & $5.3(4.2-6.4)$ & $6.1(5.1-7.2)$ & $6.4(5.5-9.0)$ & $>.05$ & .006 & .008 \\
\hline Haemoglobin (g/dl) & $14.3(13.1-15.2)$ & $13.7(12.3-14.8)$ & $13.7(12.9-14.9)$ & $14.0(13.4-15.2)$ & $>.05$ & $>.05$ & $>.05$ \\
\hline Female & $13.1(12.3-14.6)$ & $13.5(14.1-15.5)$ & $13.6(12.9-14.5)$ & $13.6(13.3-14.1)$ & $>.05$ & $>.05$ & $>.05$ \\
\hline Male & $14.8(14.1-15.5)$ & $14.1(12.7-15.2)$ & $14.4(13.0-15.0)$ & $14.6(13.4-15.9)$ & $>.05$ & $>.05$ & $>.05$ \\
\hline Platelet count, $\times 10^{3}$ platelets $/ \mathrm{mm}^{3}$ & $225(176-259)$ & $109(75-159)$ & $130(92-189)$ & $132(101-203)$ & $<.001$ & $<.001$ & $=.002$ \\
\hline Female & $244(225-259)$ & $105(75-159)$ & $113(92-213)$ & $124(101-203)$ & $<.001$ & .029 & $>.05$ \\
\hline Male & $214(167-262)$ & $111(74-157)$ & $137(94-170)$ & $153(109-227)$ & $<.001$ & $<.001$ & .004 \\
\hline ALT level (IU/ml) & $29(19-39)$ & $58(42-97)$ & $21(17-31)$ & $20(15-25)$ & $<.001$ & $<.001$ & $<.001$ \\
\hline Female & $36(18-39)$ & $69(42-106)$ & $23(17-34)$ & $21(12-31)$ & .015 & .010 & .004 \\
\hline Male & $29(19-42)$ & $57(42-81)$ & $19(17-30)$ & $20(15-25)$ & $<.001$ & $<.001$ & $<.001$ \\
\hline AST level (IU/ml) & $24(20-29)$ & $60(37-78)$ & $27(20-36)$ & $21(17-28)$ & $<.001$ & $<.001$ & $<.001$ \\
\hline Female & $27(23-37)$ & $72(42-120)$ & $30(24-36)$ & $22(17-31)$ & .004 & .003 & .004 \\
\hline Male & $23(19-29)$ & $51(37-64)$ & $24(18-35)$ & $21(16-26)$ & $<.001$ & .002 & $<.001$ \\
\hline Total bilirubin $(\mu \mathrm{mol} / \mathrm{l})$ & $9.3(6.8-12.2)$ & $14.3(10.3-21.7)$ & $10.3(7.4-17.8)$ & $9.0(7.7-10.7)$ & $<.001$ & .007 & $<.001$ \\
\hline Female & $7.2(6.2-9.3)$ & $14.3(10.3-23.2)$ & $11.3(7.4-16.0)$ & $9.7(7.8-10.7)$ & $<.001$ & .009 & .008 \\
\hline Male & $10.3(7.4-16.1)$ & $14.8(10.7-21.1)$ & $9.0(7.3-23.6)$ & $9.0(6.7-12.6)$ & .048 & $>.05$ & $<.001$ \\
\hline
\end{tabular}

attributed to these differences. For instance, the expression of NKp30 in patients with CHC may depend on the stage of liver disease. According to our research, NKp30 expression may also be related to gender diversity. In the studies mentioned above, the experimental cohorts had a different percentage of female patients $(45 \%$ and $23 \%$, respectively), which could have an impact on the results. The expression of NKG2D may be more dependent on ALT activity. As one study showed, the expression of this receptor is lower in patients with $\mathrm{CHC}$ and normal ALT activity [10]. However, we still cannot exclude the effect of other factors. 
Table 3. Median values (and interquartile range) of the NK cells' parameters measured in the healthy control group (control), in chronically HCV-infected individuals before the treatment (T0), then 6 months (T1) and one year (T2) after the beginning of DAA treatment. Underlined numbers differ significantly from those in the control group in the Mann-Whitney $U$ test. Highlighted windows present values that changed significantly after the successful treatment in the Wilcoxon signed-rank test - blue colour indicates a decrease and red means an increase

\begin{tabular}{lcccc}
\hline & Control & T0 & T1 & T2 \\
\hline NK/sample \% & $3.2(2.3-5.4)$ & $2.7(2.0-3.9)$ & $2.8(1.7-4.0)$ & $2.9(1.6-4.1)$ \\
\hline NK/lymph \% & $15(9.5-19.5)$ & $\underline{9.1(7.0-13.8)}$ & $12.7(7.3-16.8)$ & $11.2(7.0-16.0)$ \\
\hline NK GRANZ B \% & $88.6(80.9-94.2)$ & $88.4(75.2-93.9)$ & $92.7(87.0-96.2)$ & $90.1(86.7-93.8)$ \\
\hline GRANZ B MFI & $7205(4088-8714)$ & $6872(5390.5-9889.5)$ & $7109(4862.5-9137.5)$ & $6918(4169-10281)$ \\
\hline NK KIR2DS4+ \% & $1.7(0.7-6.4)$ & $\underline{6.8(1.8-29.6)}$ & $\underline{6.9(1.5-30.1)}$ & $3.1(1.4-13.9)$ \\
\hline NK KIR2DS4+ MFI & $1416(943-4148)$ & $1774(1118.5-8416)$ & $1546(988.5-4869.5)$ & $1306(858-4362)$ \\
\hline NK KIR2DL2/DL3+ \% (cd158b) & $28.1(22.3-39.1)$ & $35.8(27.5-42.8)$ & $31.1(22.2-42.3)$ & $29.5(23.6-39.5)$ \\
\hline NK KIR2DL2/DL3+ MFI & $5142(3796-5978.5)$ & $6293(4513-7851)$ & $4199(3331-6662)$ & $4668(3760.5-5795)$ \\
\hline NK NKG2A+\% & $42.0(30.0-47.4)$ & $43.7(31.5-55.3)$ & $40.4(27.9-57.3)$ & $38.8(30.4-51.7)$ \\
\hline NK NKG2A+ MFI & $6454(4514-8662)$ & $\underline{9298(7041-10959)}$ & $7328.5(5738-9156.5)$ & $5364(4784-7518)$ \\
\hline NK NKG2D+\% & $76.6(63.2-86.4)$ & $72.9(64.5-83.5)$ & $77.3(67.0-82.1)$ & $85.1(65.2-89.7)$ \\
\hline NK NKG2D+ MFI & $3033.5(2688.5-3571.5)$ & $3163(2566-3407)$ & $2958(2414-3371)$ & $2669(2284-3511)$ \\
\hline NK TRAIL+\% & $0.4(0.0-1.0)$ & $0.7(0.2-1.6)$ & $0.3(0.0-1.1)$ & $0.2(0.0-0.8)$ \\
\hline NK TRAIL+ MFI & $1172(984-1394)$ & $1093(904-1207)$ & $999(868-1377)$ & $980(851.5-2926)$ \\
\hline NK NKp30+\% & $63.9(42.3-74.2)$ & $66.6(53.5-73.5)$ & $65.3(48.9-76.1)$ & $53.9(52.2-67.4)$ \\
\hline NK NKp30+ MFI & $2958(2246-3239)$ & $\underline{3198.5(2604-3808)}$ & $2635.5(2129-3066.5)$ & $\underline{2107.5(1755.5-2435.5)}$ \\
\hline Serum CXCL10 (pg/ml) & $74.8(30.3-133.9)$ & $\underline{140.3(86.9-214.5)}$ & $91.1(53.9-146.0)$ & $64.9(34.9-127.4)$ \\
\hline
\end{tabular}

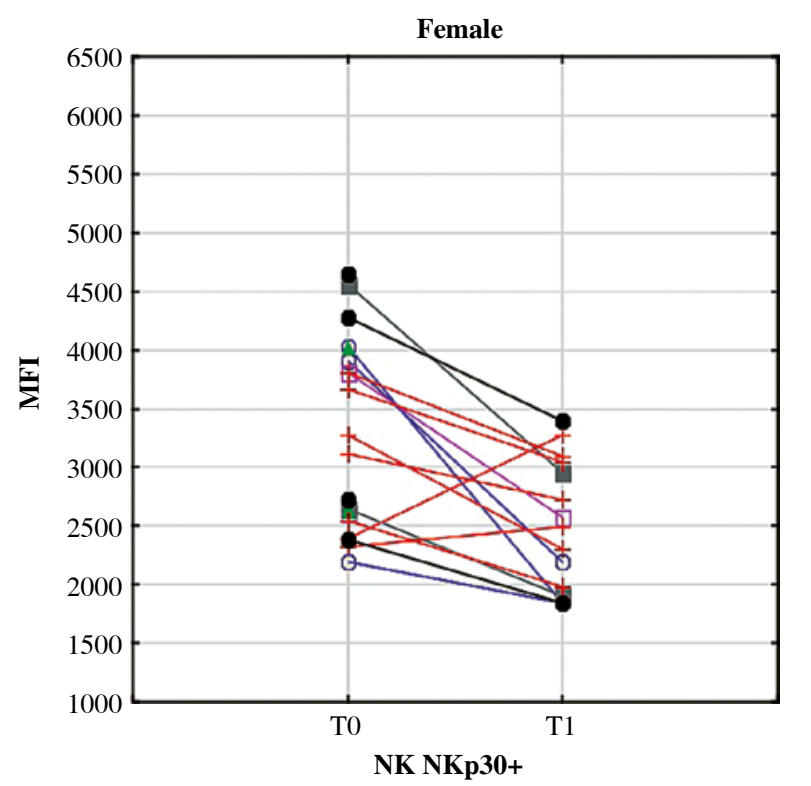

$\multimap$ GLE/PIB $\square$ GZR/EBR

$\leftarrow$ SOF/LDV $\rightarrow \mathrm{SOF} / \mathrm{LDV}+\mathrm{RBV}$

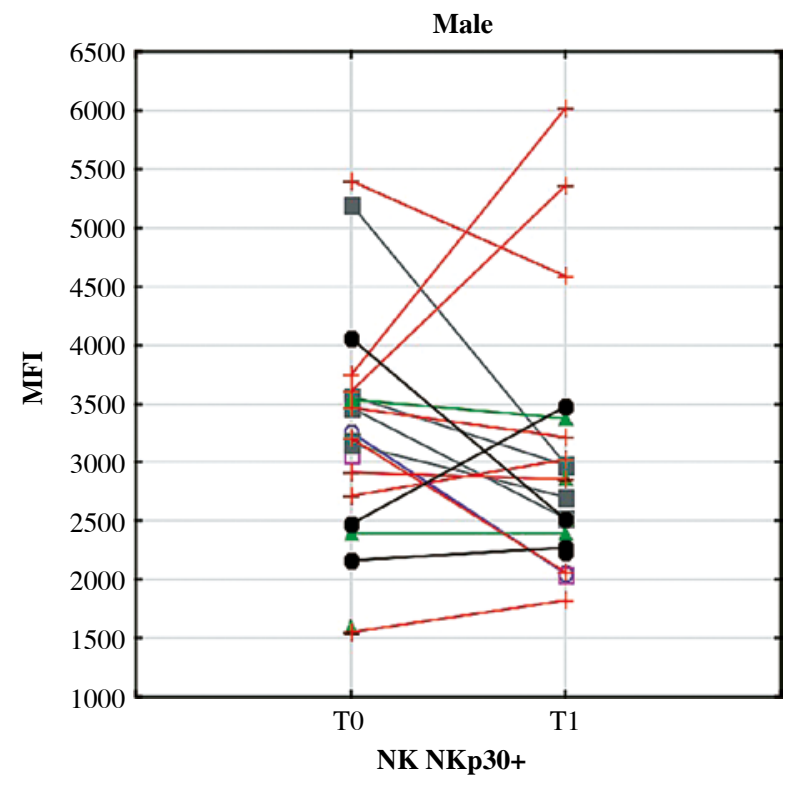

$\rightarrow \mathrm{OBV} / \mathrm{PTV} / \mathrm{r}+\mathrm{DSV}$

SOF/VEL

Fig. 2. Mean fluorescence intensity (MFI) of NKp30 on NK cells before (T0) and after (T1) the treatment in female and male patients. In the Wilcoxon signed-rank test, mean MFI in females decreased significantly $(p=0.01)$ while in males it did not change. Different colours represent treatment regimens received by each patient: GLE/PIB - glecaprevir/pibrentasvir, GZR/EBR - grazoprevir/elbasvir, OBV/PTV/r + DSV - ombitasvir/paritaprevir/ritonavir + dasabuvir, SOF/ LDV - sofosbuvir/ledipasvir, SOF/LDV + RBV - sofosbuvir/ledipasvir + ribavirin, SOF/VEL - sofosbuvirr/velpatasvir 

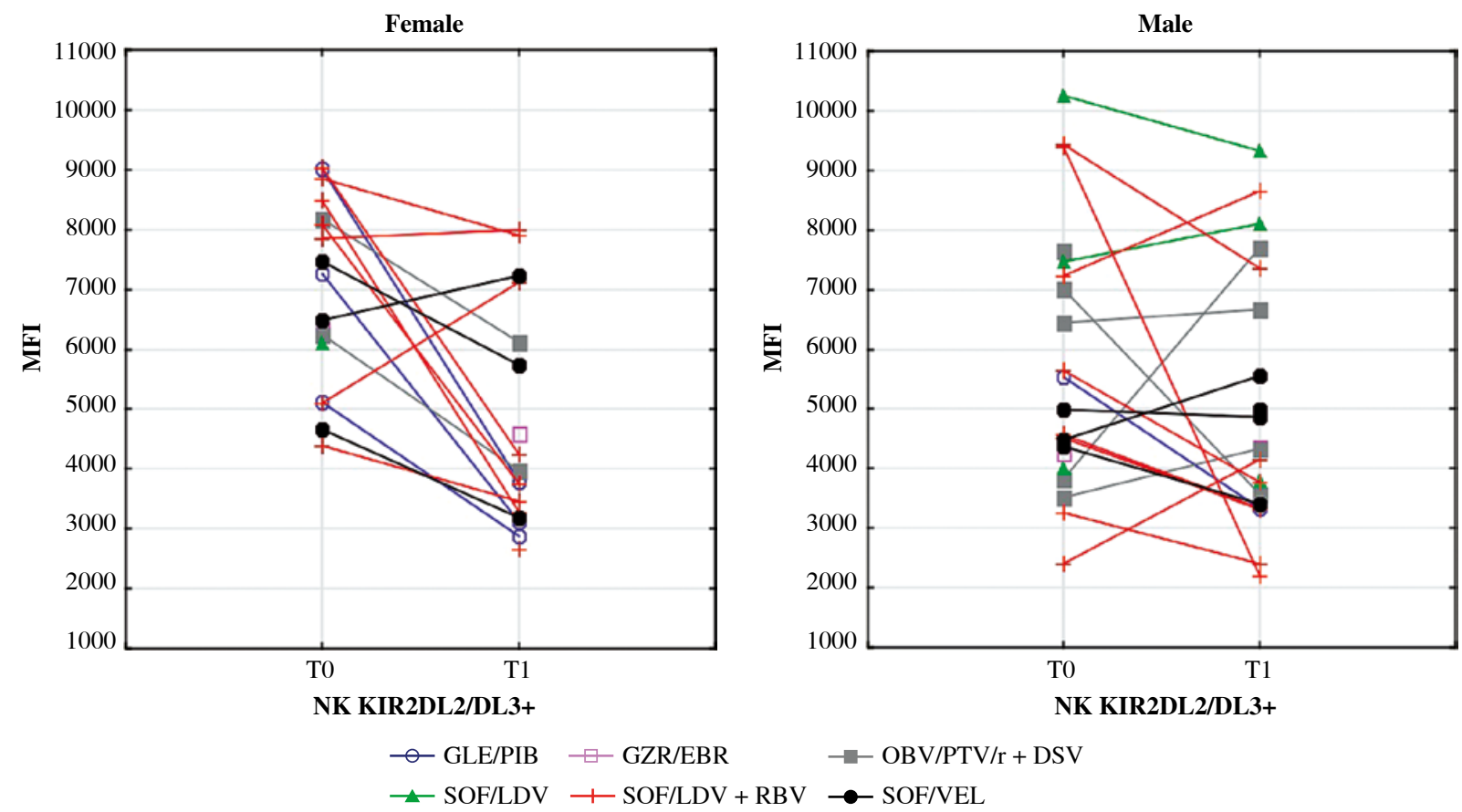

Fig. 3. Mean fluorescence intensity (MFI) of KIR2DL2/DL3 on NK cells before (T0) and after (T1) the treatment in female and male patients. In the Wilcoxon signed-rank test, mean MFI in females decreased significantly $(p=0.04)$ while in males it did not change. Different colours represent treatment regimens received by each patient: GLE/PIB - glecaprevir/ pibrentasvir, GZR/EBR - grazoprevir/elbasvir, OBV/PTV/r + DSV - ombitasvir/paritaprevir/ritonavir + dasabuvir, SOF/ LDV - sofosbuvir/ledipasvir, SOF/LDV + RBV - sofosbuvir/ledipasvir + ribavirin, SOF/VEL - sofosbuvir/velpatasvir

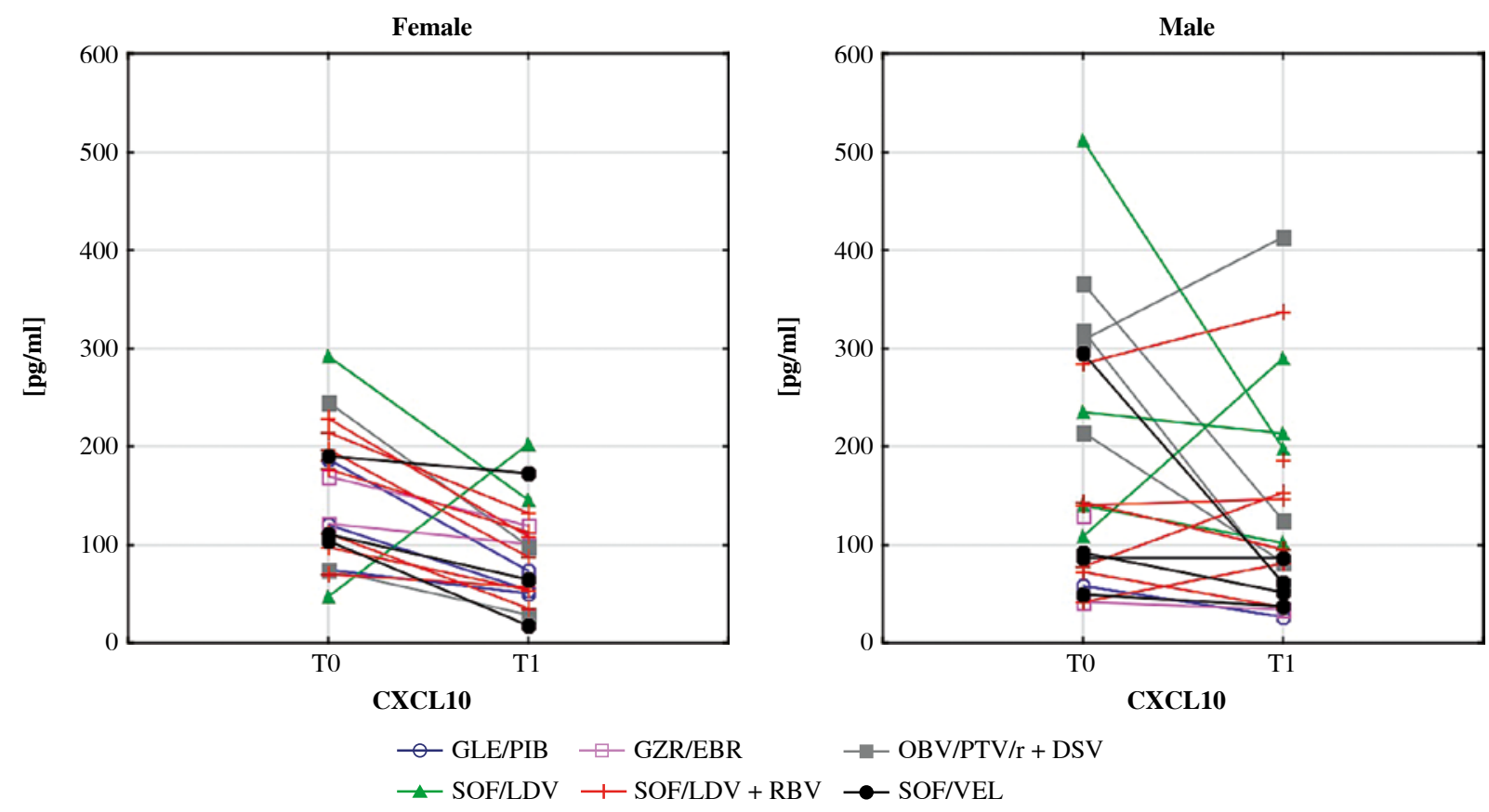

Fig. 4. Serum levels of CXCL10 before (T0) and after (T1) the treatment in female and male patients. In the Wilcoxon signed-rank test, mean concentration in females decreased significantly $(p=0.002)$ while in males it did not change. Different colours represent treatment regimens received by each patient: GLE/PIB - glecaprevir/pibrentasvir, GZR/ EBR - grazoprevir/elbasvir, OBV/PTV/r + DSV - ombitasvir/paritaprevir/ritonavir + dasabuvir, SOF/LDV - sofosbuvir/ ledipasvir, SOF/LDV + RBV - sofosbuvir/ledipasvir + ribavirin, SOF/VEL - sofosbuvir/velpatasvir 


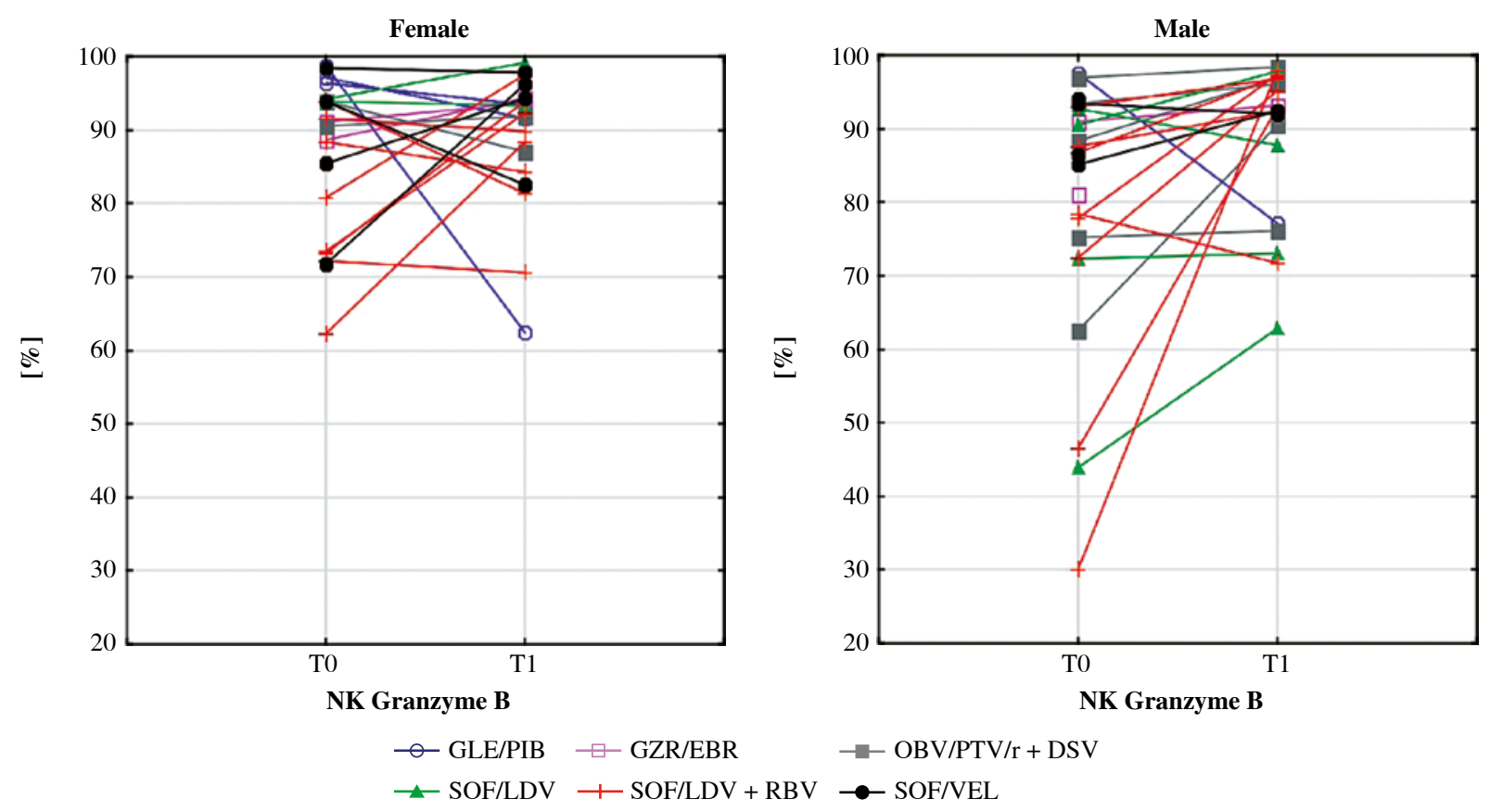

Fig. 5. Percentage of NK cells expressing granzyme B in female and male patients before the DAA treatment (T0) and after (T1). In the Wilcoxon signed-rank test mean percentage in males increased significantly $(p=0.008)$ while in females it did not change. Different colours represent treatment regimens received by each patient: GLE/PIB - glecaprevir/ pibrentasvir, GZR/EBR - grazoprevir/elbasvir, OBV/PTV/r + DSV - ombitasvir/paritaprevir/ritonavir + dasabuvir, SOF/ LDV - sofosbuvir/ledipasvir, SOF/LDV + RBV - sofosbuvir/ledipasvir + ribavirin, SOF/VEL - sofosbuvir/velpatasvir

In the current study, we observed decreased expression of the activating receptor, KIR2DS4, after the DAA treatment. This observation is highly novel. Additionally, our data describing the expression of TRAIL and serum concentrations of CXCL10 are consistent with other studies. In contrast to published studies, we observed a female-specific, post-treatment decrease of CXCL10 and a male-specific, post-treatment increase of granzyme B on NK cells. More research is required to better understand the mechanism behind these observations.

Our data showed a few differences between genders in the reaction of NK cells to the successful treatment of $\mathrm{CHC}$. $\mathrm{NKp} 30$ is an activating receptor that plays a central role in the resistance to infectious disease [33]. Its high baseline expression favours a rapid reaction of NK cells to infections such as malaria [34]. The expression of NKp30 is likely hormone-dependent as its expression has been observed to change during the different phases of the menstrual cycle [35]. In various studies, NKp30 was either increased or decreased in chronically HCV-infected patients. Its role in $\mathrm{CHC}$ disease remains elusive. One hypothesis states that in chronically infected patients, NKp30 is increased but is inactive [36]. Further, researchers have shown that NKp30 is decreased among patients who clear HCV spontaneously in the acute phase of the disease [16]. In our study, the MFI of NKp30 was increased only in chronically infected males but not in females. After treatment, NKp30 decreased significantly in women, leaving them with final values that were lower than in healthy controls. This result may indicate that the female response to infectious disease may be diminished even after successful HCV treatment.

The killer cell immunoglobulin-like receptor (KIR) repertoire on NK cells depends both on KIR and HLA gene polymorphisms [37]. Depending on the KIR genotype, male and female patients may have a different probability of becoming chronically HCV-infected [38]. Haplotype A of KIRs includes KIR2DL2/DL3 (CD158b), an inhibitory receptor that senses HLA-1 expressing cells and prevents NK cells from auto-aggression. When excessively activated, KIR2DL2/DL3 induces susceptibility to malignancies such as melanoma and its metastasis [37]. In children with CHC, KIR2DL2/DL3 is associated with more advanced liver fibrosis [39]. Additionally, there is a link between KIR2DL2 genes and HCV-related lymphoproliferative disorders [40]. The co-expression of KIR2DL2 with HLA-C1 is a significant risk factor of HCC development in HCV-infected patients [41, 42]. Some papers have reported increased expression of KIR2DL2 in chronic HCV infection, while others show that it remains unchanged. Our data indicate that the MFI of CD158b on NK cells increases in $\mathrm{HCV}$-infected females relative to healthy controls. Further, our data show that this ob- 
Table 4. Parameters of NK cells examined in the current study and assessed by other authors. Arrows indicate alterations observed among chronically HCV-infected patients and changes after the successful therapy with DAA

\begin{tabular}{|c|c|c|c|c|c|c|}
\hline \multirow[t]{2}{*}{ Parameters } & \multicolumn{2}{|c|}{ NK effector function } & \multicolumn{2}{|c|}{ Chronic HCV infection } & \multicolumn{2}{|c|}{ After DAA treatment } \\
\hline & & & In the literature & Our results & In the literature & Our results \\
\hline \multirow{2}{*}{$\begin{array}{l}\text { KIR2DS4 } \\
\text { (CD158i) }\end{array}$} & \multirow{2}{*}{$\begin{array}{l}\text { Activating } \\
\text { receptor }\end{array}$} & $\%$ & $\uparrow[5]$ & $\uparrow$ & $?$ & $\leftrightarrow($ n.s. $\downarrow)$ \\
\hline & & MFI & $?$ & $\leftrightarrow($ n.s. $\uparrow)$ & $?$ & $\downarrow$ \\
\hline \multirow[t]{2}{*}{ NKG2D } & \multirow[t]{2}{*}{$\begin{array}{l}\text { Activating } \\
\text { receptor }\end{array}$} & $\%$ & $\begin{array}{c}\uparrow[6] \\
\leftrightarrow[7-9] \\
\downarrow[10,11]\end{array}$ & $\leftrightarrow($ n.s. $\downarrow)$ & $\leftrightarrow[7,12]$ & $\uparrow$ \\
\hline & & MFI & $\begin{array}{c}\uparrow[6] \\
\downarrow[8,13]\end{array}$ & $\leftrightarrow$ (n.s. $\uparrow)$ & $\uparrow[13]$ & $\leftrightarrow$ (n.s. $\downarrow)$ \\
\hline \multirow[t]{2}{*}{ NKp30 } & \multirow[t]{2}{*}{$\begin{array}{l}\text { Activating } \\
\text { receptor }\end{array}$} & $\%$ & $\begin{array}{c}\uparrow[12,14,15] \\
\downarrow[9,16]\end{array}$ & $\leftrightarrow$ (n.s. $\uparrow)$ & $\downarrow[12,15]$ & $\leftrightarrow$ (n.s. $\downarrow)$ \\
\hline & & MFI & $\begin{array}{c}\uparrow[14,15] \\
\downarrow[9]\end{array}$ & $\begin{array}{l}\uparrow(\leftrightarrow \text { in } \\
\text { females })\end{array}$ & $\downarrow[7,13,15]$ & $\downarrow(\leftrightarrow$ in males $)$ \\
\hline \multirow{2}{*}{$\begin{array}{l}\text { KIR2DL2/ } \\
\text { DL3 } \\
\text { (CD1586) }\end{array}$} & \multirow{2}{*}{$\begin{array}{l}\text { Inhibiting } \\
\text { receptor }\end{array}$} & $\%$ & $\uparrow[10,15]$ & $\leftrightarrow$ (n.s. $\uparrow)$ & $\downarrow[15]$ & $\leftrightarrow($ n.s. $\downarrow)$ \\
\hline & & MFI & $\uparrow[16]$ & $\begin{array}{l}\leftrightarrow(\uparrow \text { in } \\
\text { females })\end{array}$ & $?$ & $\downarrow(\leftrightarrow$ in males $)$ \\
\hline \multirow[t]{2}{*}{ NKG2A } & \multirow{2}{*}{$\begin{array}{l}\text { Inhibiting } \\
\text { receptor }\end{array}$} & $\%$ & $\uparrow[9,15,17]$ & $\leftrightarrow$ (n.s. $\uparrow)$ & $\leftrightarrow[15]$ & $\leftrightarrow($ n.s. $\downarrow)$ \\
\hline & & MFI & $\uparrow[7,15,17]$ & $\uparrow$ & $\downarrow[7,15]$ & $\downarrow$ \\
\hline \multirow[t]{2}{*}{ TRAIL } & \multirow[t]{2}{*}{$\begin{array}{c}\text { Direct } \\
\text { cytotoxicity }\end{array}$} & $\%$ & $\begin{array}{l}\uparrow[7] \\
\uparrow[17]\end{array}$ & $\leftrightarrow$ (n.s. $\uparrow)$ & $\downarrow[7,12]$ & $\downarrow$ \\
\hline & & MFI & $\begin{array}{l}\overleftrightarrow{\leftrightarrow}[7] \\
\uparrow[17]\end{array}$ & $\leftrightarrow$ & $\downarrow[7]$ & $\leftrightarrow($ n.s. $\downarrow)$ \\
\hline \multirow[t]{2}{*}{ Granzyme B } & \multirow[t]{2}{*}{$\begin{array}{l}\text { Cytotoxic } \\
\text { granules }\end{array}$} & $\%$ & $?$ & $\leftrightarrow$ & $?$ & $\begin{array}{l}\uparrow(\leftrightarrow \text { in } \\
\text { females })\end{array}$ \\
\hline & & MFI & $\uparrow[13]$ & $\leftrightarrow$ & $\downarrow[13]$ & $\leftrightarrow$ \\
\hline CXCL10 & Chemokine & & $\uparrow[7]$ & $\uparrow$ & $\downarrow[7]$ & $\downarrow(\leftrightarrow$ in males $)$ \\
\hline
\end{tabular}

MFI - mean fluorescence intensity, n.s. - statistically non-significant

servation normalises after successful treatment. In males, the expression of that receptor remained unchanged in all examined groups, regardless of the infection status.

Activation-induced NK cell death is accompanied by the leakage of granzyme B from intracellular granules into the cytoplasm. This serine protease contributes to targeted cell death, playing an important role in the development of autoimmunity, e.g., rheumatoid arthritis [43]. The T-cells of patients with $\mathrm{HCC}$ have a diminished ability to produce granzyme B [44]. Due to its antigen-specific properties, this protease is considered a novel therapy for solid tumours [45]. In our study, the percentage of NK cells expressing granzyme B did not differ between chronically $\mathrm{HCV}$-infected patients and controls but was increased in treated men relative to women. This finding may suggest that males are better protected by NK cells against HCC and autoimmunity after therapy. Since the occurrence and recurrence rates of $\mathrm{HCC}$ are higher among men, the immunological mechanisms of this phenomenon require further investigation [26].

CXCL10 is a chemokine produced by hepatocytes and liver-infiltrating lymphocytes during HCV infection.
It is well understood that CXCL10 induces the migration of $\mathrm{T}$ cells and NK cells to the liver [46]. High levels of CXCL10 are correlated with poor treatment outcomes in patients with chronic hepatitis $\mathrm{C}$ and decompensated cirrhosis treated with DAA [47]. Furthermore, elevated serum concentrations of this chemoattractant are associated with HCV-related mixed cryoglobulinaemia, especially in patients with clinically active vasculitis [48]. Female gender is one of the main factors related to cryoglobulin production [49]. Interferon-free therapies seem to decrease vasculitis in most HCV patients [50, 51]. In our study, CXCL10 levels were reduced in women after therapy. This observation confirms the significant role of CXCL10 in the pathogenesis of $\mathrm{HCV}$-related mixed cryoglobulinemia. CXCL10 is speculated to be a candidate for a novel therapeutic target in this condition [48]. Our study supports this concept.

In conclusion, post-menopausal females and males differ in the reaction of their NK cells to interferon-free therapy of CHC. Our research may lead to more studies on the different nature of female and male immune systems in the context of HCV infection and treatment. 


\section{Acknowledgements}

This work was supported by the National Science Centre, Poland (NCN) grant No. 2016/23/B/NZ6/01497.

\section{The authors declare no conflict of interest.}

\section{References}

1. Prager I, Watzl C (2019): Mechanisms of natural killer cell-mediated cellular cytotoxicity. J Leukoc Biol 105: 13191329.

2. Abel AM, Yang C, Thakar MS, et al. (2018): Natural killer cells: Development, maturation, and clinical utilization. Front Immunol 9: 1869.

3. Yoon JC, Yang CM, Song Y, et al. (2016): Natural killer cells in hepatitis C: Current progress. World J Gastroenterol 22: 1449-1460.

4. Castello G, Scala S, Palmieri G, et al. (2010): HCV-related hepatocellular carcinoma: From chronic inflammation to cancer. Clin Immunol 134: 237-250.

5. Podhorzer A, Dirchwolf M, Machicote A, et al. (2018): The clinical features of patients with chronic hepatitis $C$ virus infections are associated with killer cell immunoglobulin-like receptor genes and their expression on the surface of natural killer cells. Front Immunol 8: 1912.

6. Oliviero B, Varchetta S, Paudice E, et al. (2009): Natural killer cell functional dichotomy in chronic hepatitis $\mathrm{B}$ and chronic hepatitis C virus infections. Gastroenterology 137: 1151-1160.

7. Serti E, Chepa-Lotrea X, Kim YJ, et al. (2015): Successful interferon-free therapy of chronic hepatitis $\mathrm{C}$ virus infection normalizes natural killer cell function. Gastroenterology 149: 190-200.e2.

8. Séne D, Levasseur F, Abel M, et al. (2010): Hepatitis C virus (HCV) evades NKG2D-dependent NK cell responses through NS5A-mediated imbalance of inflammatory cytokines. PLoS Pathog 6: e1001184.

9. Nattermann J, Feldmann G, Ahlenstiel G, et al. (2006): Surface expression and cytolytic function of natural killer cell receptors is altered in chronic hepatitis C. Gut 55: 869-877.

10. Szereday L, Meggyes M, Halasz M, et al. (2016): Immunological changes in different patient populations with chronic hepatitis C virus infection. World J Gastroenterol 22: 4848-4859.

11. Dessouki O, Kamiya Y, Nagahama H, et al. (2010): Chronic hepatitis $\mathrm{C}$ viral infection reduces NK cell frequency and suppresses cytokine secretion: Reversion by anti-viral treatment. Biochem Biophys Res Commun 393: 331-337.

12. Spaan M, van Oord G, Kreefft K, et al. (2016): Immunological analysis during interferon-free therapy for chronic hepatitis $\mathrm{C}$ virus infection reveals modulation of the natural killer cell compartment. J Infect Dis 213: 216-223.

13. Strunz B, Hengst J, Deterding K, et al. (2018): Chronic hepatitis $C$ virus infection irreversibly impacts human natural killer cell repertoire diversity. Nat Commun 9: 2275.

14. De Maria A, Fogli M, Mazza S, et al. (2007): Increased natural cytotoxicity receptor expression and relevant IL-10 production in NK cells from chronically infected viremic HCV patients. Eur J Immunol 37: 445-455.

15 Perpinán E, Pérez-Del-Pulgar S, Londońo MC, et al. Cirrhosis Hampers Early and Rapid Normalization of Natural Killer
Cell Phenotype and Function in Hepatitis C Patients Undergoing Interferon-Free Therapy. Front Immunol; 11. Epub ahead of print 25 February 2020. DOI: 10.3389/fimmu.2020.00129.

16. Ji HF, Wang J, Yu L, et al. (2013): High frequencies of $\mathrm{CD} 158 \mathrm{~b}+\mathrm{NK}$ cells are associated with persistent hepatitis $\mathrm{C}$ virus infections. Ann Hepatol 12: 371-379.

17. Ahlenstiel G, Titerence RH, Koh C, et al. (2010): Natural killer cells are polarized toward cytotoxicity in chronic hepatitis $\mathrm{C}$ in an interferon-alfa-dependent manner. Gastroenterology 138: 325-335.e1-2.

18. Jaillon S, Berthenet K, Garlanda C (2019): Sexual dimorphism in innate immunity. Clin Rev Allergy Immunol 56: 308-321.

19. Phan MT, Chun S, Kim SH, et al. (2017): Natural killer cell subsets and receptor expression in peripheral blood mononuclear cells of a healthy Korean population: Reference range, influence of age and sex, and correlation between NK cell receptors and cytotoxicity. Hum Immunol 78: 103-112.

20. Giefing-Kröll C, Berger P, Lepperdinger G, et al. (2015): How sex and age affect immune responses, susceptibility to infections, and response to vaccination. Aging Cell 14: 309-321.

21. Al-Attar A, Presnell SR, Peterson CA, et al. (2016): The effect of sex on immune cells in healthy aging: Elderly women have more robust natural killer lymphocytes than do elderly men. Mech Ageing Dev 156: 25-33.

22. Jilkova ZM, Decaens T, Marlu A, et al. (2017): Sex differences in spontaneous degranulation activity of intrahepatic natural killer cells during chronic hepatitis B: Association with estradiol levels. Mediators Inflamm 2017: 3214917.

23. Ruggieri A, Gagliardi MC, Anticoli S (2018): Sex-dependent outcome of hepatitis B and C Viruses infections: Synergy of sex hormones and immune responses? Front Immunol 9: 2302.

24. Ruggieri A, Barbati C, Malorni W (2010): Cellular and molecular mechanisms involved in hepatocellular carcinoma gender disparity. Int J Cancer 127: 499-504.

25. Mozer-Lisewska I, Zwolińska K, Kowala-Piaskowska AE, et al. (2016): Genetic (KIR, HLA-C) and some clinical parameters influencing the level of liver enzymes and early virologic response in patients with chronic hepatitis C. Arch Immunol Ther Exp (Warsz) 64: 65-73.

26. Rinaldi L, Perrella A, Guarino M, et al. (2019): Incidence and risk factors of early $\mathrm{HCC}$ occurrence in $\mathrm{HCV}$ patients treated with direct acting antivirals: a prospective multicentre study. J Transl Med 17: 292.

27. Halota W, Flisiak R, Juszczyk J, et al. (2018): Rekomendacje Polskiej Grupy Ekspertów HCV dotyczące leczenia wirusowych zapaleń wątroby typu C w roku 2018. Hepatologia 18: $1-9$.

28. Jung KS, Kim SU (2012): Clinical applications of transient elastography. Clin Mol Hepatol 18: 163.

29. Kierepa A, Witkowska A, Kaczmarek M, et al. (2020): Impact of chronic HCV treatment on quality of life of patients with metabolic disorders in context of immunological disturbances. Sci Rep 10: 1-10.

30. Skinner J (2018): Statistics for Immunologists. Curr Protoc Immunol 122: 54.

31. Hsu WF, Lai HC, Su WP, et al. (2019): Rapid decline of noninvasive fibrosis index values in patients with hepatitis $\mathrm{C}$ receiving treatment with direct-acting antiviral agents. BMC Gastroenterol 19: 63.

32. Horowitz A, Strauss-Albee DM, Leipold M, et al. (2013): Genetic and environmental determinants of human NK cell 
diversity revealed by mass cytometry. Sci Transl Med 5: $208 \mathrm{ra} 145$.

33. Barrow AD, Martin CJ, Colonna M (2019): The natural cytotoxicity receptors in health and disease. Front Immunol 10: 909.

34. Walk J, Sauerwein RW (2019): Activatory receptor NKp30 predicts NK cell activation during controlled human malaria infection. Front Immunol 10: 2864.

35. Ponnampalam AP, Gargett CE, Rogers PAW (2008): Identification and hormonal regulation of a novel form of NKp30 in human endometrial epithelium. Eur J Immunol 38: 216-226.

36. Marras F, Bozzano F, Ascierto ML, et al. (2014): Baseline and dynamic expression of activating $\mathrm{NK}$ cell receptors in the control of chronic viral infections: The paradigm of HIV-1 and HCV. Front Immunol 5: 305.

37. Konjevic G, Jurisic V, Jovic V, et al. (2012): Investigation of NK cell function and their modulation in different malignancies. Immunol Res 52: 139-156.

38. Kuśnierczyk P, Mozer-Lisewska I, Zwolińska K, et al. (2015): Contribution of genes for killer cell immunoglobulin-like receptors (KIR) to the susceptibility to chronic hepatitis $\mathrm{C}$ virus infection and to viremia. Hum Immunol 76: 102-108.

39. Mania A, Kaczmarek M, Kemnitz P, et al. (2017): Expression of natural killer cell inhibitory receptors is associated with significant liver injury in chronic hepatitis $\mathrm{C}$ in children. Ann Hepatol 16: 521-529.

40. De Re V, Caggiari L, De Zorzi M, et al. (2015): Genetic diversity of the KIR/HLA system and susceptibility to hepatitis C virus-related diseases. PLoS One 10: e0117420.

41. Saito H, Umemura T, Joshita S, et al. (2018): KIR2DL2 combined with HLA-C1 confers risk of hepatitis $\mathrm{C}$ virusrelated hepatocellular carcinoma in younger patients. Oncotarget 9: 19650-19661.

42. Cariani E, Pilli M, Zerbini A, et al. (2013): HLA and killer immunoglobulin-like receptor genes as outcome predictors of hepatitis $\mathrm{C}$ virus-related hepatocellular carcinoma. Clin Cancer Res 19: 5465-5473.

43. Ida H, Utz PJ, Anderson P, et al. (2005): Granzyme B and natural killer (NK) cell death. Mod Rheumatol 2005; 15: 315-322.

44. Kalathil S, Lugade AA, Miller A, et al. (2013): Higher frequencies of GARP+CTLA-4+Foxp3+ t regulatory cells and myeloid-derived suppressor cells in hepatocellular carcinoma patients are associated with impaired T-cell functionality. Cancer Res 73: 2435-2444.

45. Qian X, Shi Z, Qi H, et al. (2019): A novel Granzyme B nanoparticle delivery system simulates immune cell functions for suppression of solid tumors. Theranostics 9: 7616-7627.

46. Riva A, Laird M, Casrouge A, et al. (2014): Truncated CXCL10 is associated with failure to achieve spontaneous clearance of acute hepatitis $\mathrm{C}$ infection. Hepatology 60 : 487-496.

47. Childs K, Merritt E, Considine A, et al. (2017): Immunological predictors of nonresponse to directly acting antiviral therapy in patients with chronic hepatitis $\mathrm{C}$ and decompensated cirrhosis. Open Forum Infect Dis 4: ofx067.

48. Mazzi V, Ferrari SM, Giuggioli D, et al. (2015): Role of CXCL10 in cryoglobulinaemia. Clin Exp Rheumatol 33: 433436.

49. Cacoub P, Saadoun D (2008): Hepatitis C virus infection induced vasculitis. Clin Rev Allergy Immunol 35: 30-39.

50. Mazzaro C, Dal Maso L, Quartuccio L, et al. (2018): Longterm effects of the new direct antiviral agents (DAAs) therapy for HCV-related mixed cryoglobulinaemia without renal in- volvement: a multicentre open-label study. Clin Exp Rheumatol 36 Suppl 111: 107-114.

51. Sise ME, Bloom AK, Wisocky J, et al. (2016): Treatment of hepatitis $\mathrm{C}$ virus-associated mixed cryoglobulinemia with direct-acting antiviral agents. Hepatology 63: 408-417. 